\title{
Understanding significance of sitting posture as a etiological factor causing low back pain
}

\author{
Rajan Chaudhari*1, Santosh Chavhan ${ }^{2}$
}

1. PG Scholar (Rognidan \& Vikrutti Vidynan),

2. MD (Ayu) HOD and Professor,

Dept. of Rognidan \& Vikrutti Vidynan, School of Ayurveda,

D.Y.Patil Univeristy, Nerul, Navi Mumbai Maharashtra, India

*Corresponding author: Email id- dr.rajanchaudhari1992@gmail.com

\begin{abstract}
Good posture is important because it minimizes the excessive force that muscles and joints need to absorb. Psychology studies have suggested that slouchier postures make people look less confident to others, and even feel less competent, Henderzahs-Mason said. There's also some evidence showing that people who walk with a slouched posture have a higher predisposition to depression than those who walk more upright. In this article we have tried to compile the significance of sitting posture as a etiological factor causing low back pain. Back pain has been termed as "an illness in search of a disease".
\end{abstract}

Keywords: Sitting posture, low back pain

\section{INTRODUCTION}

Good posture is also known as neutral spine. When we have good posture, the muscles surrounding the spine are balanced and supporting the body equally .There are plenty of reasons to maintain good posture, and doing so can benefit a person's body and mind. The main health risks of poor posture are aches and pains, which can occur right away or down the road, said Jill Henderzahs-Mason, a wellness physical therapist at the Mayo Clinic's Healthy Living Program in Rochester, Minnesota. This discomfort may start in the neck or back, but ultimately, it may affect the hips or knees, she said.

People who exhibit bad posture also may be less efficient when they move, Henderzahs-Mason said. "They don't move as well or as fast, and are predisposed to injury," she said. Posture in which the shoulders are hunched forward can even 
impact breathing, because the diaphragm and the lungs have less room to expand, and this narrows the airways.

Posture: Posture is a "position or attitude of the body a relative arrangement of body part for a specific activity or a characteristic manner of bearing the body. Posture refers to the preferred biomechanical alignment of the body, said Eric Robertson, director of graduate physical therapy education at Kaiser Permanente of Northern California and a spokesperson for the American Physical Therapy Association.

\section{Classification of posture}

\section{A. Inactive Posture \\ B. Active -i) Static Posture ii) Dynamic posture}

\section{Concept of good/correct posture:}

Good / Correct Posture-Good posture is the state of muscular and skeletal balance that protect the supporting structures of the body against injury or progressive deformity irrespective of the attitude. Opportunity for plenty of natural free movement.

Concept of Faulty Posture: any position that increases stress on joints is faulty posture.

\section{Positional/Postural}

2. Structural

Faulty posture analysis

1. Lordotic posture

2. Kyphotic posture / Round back

3. Scoliotic posture

\section{Sway back posture / slouched posture}

\section{Flat back posture}

\section{Flat neck posture}

\section{Forward head posture}

\section{Types of Faulty Posture}

(1) Lordotic Posture - Lordosis is the normal curve (anterior convexity) of cervical and lumbar spine which is found all normal individual pathologically. It is an exaggeration of the normal curve found in cervical and lumbar spine.

- Potential Sources of Pain: Stress to the anterior longitudinal ligament, Narrowing of the posterior disk space and narrowing of intervertebral foramen, Approximation of the articular facets. The facets may become weight bearing which may cause syonovial irritation and joint inflammation.

- Common Cause of Excessive Lumbar Lordosis :Weakness of abdominal muscle, Tightness or contracture of hip flexor (iliopsoas), Congenital problems such as bilateral congenital dislocation of hip ,Pregnancy, High heel shoes / foot wears, Spondylolisthesis, Anterior tilt of pelvis as a result of weak extensor of hip and Abdominals ,Tightness or shortening of cervical extensor.

- Treatment for Excessive Lumbar Lordosis

1. Mobilization of the lumbar spine. 
2. Anterior stretching of the lumbar spine

3. Strengthening of the abdominals, glutei and hamstring.

4. Training in grade correction of pelvic tilt has to be emphasized active backward or posterior pelvic tilting by contracting abdominals and glutei in supine is initiated.

5. Toe touching in long sitting or forward bending sitting exercise

6. Spinal extension or hyper extension should be strictly avoided.

_(2) Kyphotic Posture / Round Back - It is a faulty posture in which lumbar spine and cervical spine get hyper extended while thoracic spine get flexed and head become slightly forward.

- Potential Sources of Pain: Stressed to the posterior longitudinal ligament,Fatigue of the thoracic erector spinae and rhomboid muscle,Thoracic outlet syndromes,Cervical posture syndromes.

- Common Cause of kyphosis $\neg$ shortening or tightness of extensors of cervical spine and lumbar spine and flexor of hip joint. $\neg$ Weakness of neck flexors,upper back extensors (erector spinae) and Hamstring muscle. $\neg$ Bony anomaly generally in anterior tilt of pelvis, abdominals get elongated but in this posture excessive flexion of thoracic spine offsets the effect of anterior pelvic tilt. $\neg$ Ankylosing spondylitis. $\neg$ Other congenital anomalies.

- _Treatment of kyphosis: Relaxation ,Repeated stretching session ,Mobilization of the whole spine, Resistive exercise for longitudinal and transverse back muscle ,Controlled pelvic tilt

(3) Scoliotic Posture- A lateral curvature of spine which exceeds $10^{\circ}$ bending of the vertebral from the normal is tended as scoliosis column to one side combined with rotation of the vertebral bodies towards the convexity and the spinous process towards the concavity.

- Potential Source of Pain -Muscle fatigue and ligamentous strain on the side of convexity, Nerve root irritation on the side of concavity.

- Common Cause: Structural scoliosis - Neuromuscular disease, osteopathic disorder, and idiopathic disorder $\neg$ Non structural - Leg length discrepancy, either structural or functional, muscle guarding or spasm a painful stimuli in the back or neck, and habitual or asymmetric posture.

- Treatment of scoliosis: Active Correction with postural adaptation ,Passive Correction by Hanging, Educate the patient by active effort ,Relaxation technique, Repeated sessions of maintenance ,General free mobility exercises ,Deep breathing ,Balance Exercises, Traction,CCP - Curve correcting pad Milwaukee brace 
(4) Sway Back Posture/Slouched - It is faulty posture in which head becomes slightly forward there is extension of cervical spine, flexion of thoracic and loss of lordosis of lumbar spine extension of hip and knee joint during standing are also the feature of sway back posture pelvis rotates posterior. In this there is increased pelvic inclination up to $40^{\circ}$. When standing for prolonged period the person usually assumes an asymmetric stance. In which most of the weight is borne on one lower extremity with periodic shifting of weight to the opposite extremity.

- Potential Source of Pain - Stress to iliofemoral ligament, the anterior longitudinal ligament of lower lumbar spine and posterior longitudinal ligament of upper lumbar and thoracic spine.Narrowing of intervertebral foramen in lower lumbar spine that may compress the blood vessel dura \& nerve root. Approximation of articular facets in to lower lumbar spine

- Common Cause of Sway Back

1) Tightness of hamstring and abdominal muscle. 2) Weakness of one joint iliopsoas 3) Bony anomaly

- Treatment of Sway Back :Stretching of hamstring and abdominal muscle, Relaxation of the body, Strengthening of iliopsoas, Maintain position of head is backward, extension of thoracic Spine, Maintain normal lordosis of lumbar spine, Always standing in erect position
5) Flat Back Posture - Flat back is faulty posture in which whole lumbar and thoracic spine gets flattened. Although the cause and symptom of both flat back and sway back are common but can be differentiated by excessive flexion and back ward deviation of the upper thoracic spine in sway back posture while in flat back posture spine become almost straight. It is reverse a lumbar lordosis. There is flattening of normal lumbar lordosis.

- Potential Source of Pain: Lack of the normal physiologic lumbar curve which reduces the shock absorbing effect of lumbar region and predisposes the person to injury. Stress to the posterior longitudinal ligament.Increase of the posterior disk space which allow the nucleus pulposus to imbibe extra fluid and under certain circumstance may protrude posteriorly when the person attempts extension.

- Common Cause of Flat Back 1) Tight trunk flexor (rectus abdominis and intercostal) and hip extensor muscle. 2) Stretched and weak lumbar extensor and possibly hip flexor muscle.

- Treatment of Flat Back: Increase lumbar lordosis which results in forward tilting of pelvis. • Maintained of arch by active holding and also passive support in sitting are effective in maintaining lordosis. - Mobility and strengthening exercise of lumbar extensor are important. - Stretching of trunk flexor and hip extensor muscle. 
6) Flat Neck Posture - It is an abnormal posture which is characterized by any increased upper flexion of the occiput on atlas and decreased lordosis of the cervical spine. It may be seen with an exaggerated military posture. There may be tempomandibular joint dysfunction with protection of the mandible.

- Potential Source of Pain: Temporomanibular joint pain and occlusive changes, Decrease in the shock absorbing function of the lordotic curve which may predispose the neck to injury, Stress to ligamentum nuchae.

- Common Cause of Flat Neck Posture: Short anterior neck muscle .Activity which require straightening of cervical spine predisposes to this type of posture such as - soldier keep their upper back straight(attention position) for prolonged period of time, using high pillow under the head and spasm of cervical spine.

- Treatment of Flat Neck Posture: Relaxed passive movement this includes manipulation and mobilization of cervical spine. Strong isometric are indicated when mobility is contraindicated but strength, endurance and tone of the cervical muscle are maintained or improved. Stretching the anterior neck muscle. Strengthening exercises of levator scapulae, strenocledomastoid and scalene muscle. Improvement of the posture and function of neck.

7) Forward Head Posture - It is faulty posture which is characterized by excessive extension of upper cervical spine and flexion of the lower cervical and upper thoracic. There also may be temporomandibular Joint (TMJ) dysfunction with retrusion of the mandible.

- Potential Source of Pain: Stress to anterior longitudinal ligament in the upper cervical spine and posterior longitudinal ligament in the lower cervical and upper thoracic spine. Muscle tension or fatigue. Irritation of facet joint in upper cervical spine Narrowing of the intervertebral foramina in the upper cervical region which may impinge on the blood vessel and nerve roots, especially if there are degenerative change

- Impingement on the neurovascular bundle from anterior scalene muscle tightness. Impingement on the cervical plexus from levator scapulae muscle tightness. Impingement on the greater occipital nerve from a tight or tense upper trapezius muscle leading to tension headaches.TMJ pain from faulty head, neck and manibular alignment and associated facial muscle tension. Lower cervical disc lesion from the faulty flexed posture.

- Cause of Forward Head Posture: Working on computer which is slightly higher than the position of head. Enthusiastically watching match on television for prolonged time also predisposes to this type of faulty posture. Using of high pillow under the neck.Tight levator scapulae,Sternocledomastoid,Scale 
ne and Sub-occipital muscle. Stretched and weakened anterior throat muscle and lower cervical and upper thoracic erector spinae muscle.

- Treatment of Forward Head Posture: Stretching of levator scapulae, Sterocledomastoid, Scalene and Sub-occipital muscle. Avoid pillow or small pillow under the neck. PNF (Proprioceptive Neuromuscular Fasciculation) technique ideally combines all the four above mentioned effect selectively. Strong isometric and indicated when mobility is contraindicated but strength, endurance and tone of the cervical muscle are to be maintained or improved.

\section{DISCUSSION}

- Twist your torso to activate your side abs: Strengthens obliques so the right muscles are activated when sitting or standing.

- When bones and joints are in correct alignment, it allows the muscles to be used as they're intended, and have less fatigue and more energy, explains Griffith. In other words, "the muscles don't have to work so hard to do what they're supposed to do.

- When sitting, feet should rest flat on the floor, with even weight on both hips. Back should be mostly straight (have natural curves in lumbar, thoracic, and cervical areas).shoulders should be back but relaxed and ears should line up over collarbones.

- When standing, legs should have a slight knee bend and not hyper extending or locking knee joints.

- Postural muscles should get break once in a while. They can get overworked and cause pain.

- Poor posture can contribute to tension headaches, due to increased muscle tension in the back of the neck. Often if we correct our posture, we can reduce muscle tension and improve our headaches.

- A physical therapist can evaluate someone's posture to identify what is out of balance and what might be the cause.

\section{REFERENCES}

1. Andersson GB. Epidemiological features of chronic low pain. Lancet. 1999;354:581-5. doi: 10.1016/S0140-6736(99)01312[PubMed] [CrossRef] [Google Scholar]

2. Dillane J, Fry J, Kalton G. Acute back syndrome - a study from general practice. $\mathrm{Br}$ Med J. 1966;2:82-4. doi: 10.1136/bmj.2.5505.82. [PMC free article] [PubMed] [CrossRef] [Google Scholar]

3. Andersson HI, Ejlertsson G, Leden I, et al. Chronic pain in a geographically defined general population: studies of differences in age, gender, social class and pain localization. Clin J 
Pain. 1993;9:174-

82. [PubMed] [Google Scholar]

4. Andersson GB. The epidemiology of spinal disorders. In: Frymoyer JW, editor. The adult spine: principles and practice. 2. Philadelphia, PA: LippincottRaven; 1997. [Google Scholar]

5. Tulder MW, Koes BW, Bouter LM. A cost-of-illness study of back pain in The Netherlands. Pain. 1995;62:233$40 . \quad$ doi: 10.1016/03043959(94)00272-G. [PubMed] [CrossRef] [Google Scholar]

6. Deyo R, Cherkin D, Conrad D. Cost, controversy, crisis: low back pain and the health of the public. Annu Rev Publ Health. 1991;12:141-56. doi: 10.1146/annurev.pu.12.050191.00 1041. [PubMed]

[CrossRef] [Google Scholar]

7. Bogduk N. The innervation of the lumbar spine. Spine. 1983;8:28693. doi: 10.1097/00007632198304000-00009. [PubMed] [CrossRef] [Google Scholar]

8. Schneck CD. The anatomy of lumbar spondylosis. Clin Orthop Relat Res. 1985;193:2036. [PubMed] [Google Scholar]

"An overlook on various causes of irritable bowel syndrome (IBS)."

Ratnaraj Patil, Sachin Naik

Ayurline: International Journal of Research In Indian Medicine 2020;4(5):01- 07 